\title{
Mathematical approach to human character
}

\author{
2020/11/24 First Edition \\ Takuya Yabu(takuya.yabu@live.jp)
}

\begin{abstract}
I thought about whether to receive positive or negative emotions from an event from the perspective of human character. Regarding the human character, I define it as a process of selecting one's emotion $x$ so that the received emotion $x$ becomes $x=0$ with respect to the event $X$ and the reaction of the other party when one's thoughts and reactions occur as the accompanying reactions. Mathematically modeled it, the probability density function of how much to select an emotion has a fixed probability distribution. I also described how to deal with one's character as an application of this model.
\end{abstract}

\section{Introduction}

I wrote a paper about human emotion processing (Yabu, 2020). I have shown that for an individual, there is a fixed probability distribution function that receives emotions from events, and a fixed distribution function that also has a response from the received emotions. It was also found that the group of individuals is also represented by a fixed distribution of emotions and reactions as a group. Although it is now possible to explain emotion processing using mathematical models, it remains unclear whether individuals or groups adopt positive or negative emotions when receiving emotions from events. I think this is the part of the human character.

So, I mathematically thought about how emotional choices are made according to human character and how I should deal with it because of my negative and disliked character.

2. About individual's character

2.1. What is an individual's character?

In the paper I wrote (Yabu, 2020), I described human emotion processing using a mathematical model that receives emotions $x$ from events $X$ and responds $F(x)$ to those emotions $x$ received. Figure 1 shows the relationship. 


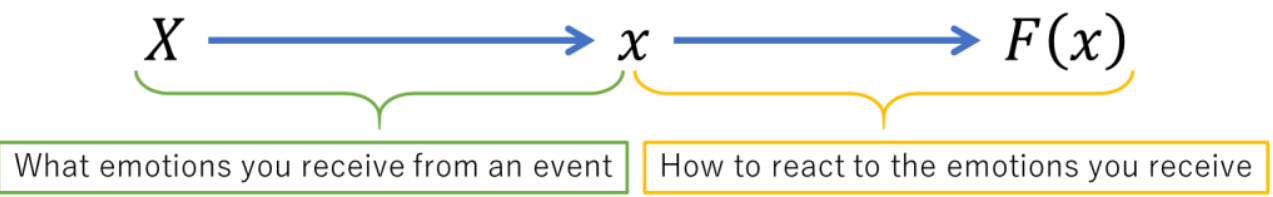

Figure 1 About the relationship between events, emotions, and reactions

In my mathematical model, the emotion $x$ was determined from the following relationship between the event and the reaction function. However, I did not say whether to choose positive or negative emotions.

$$
X=F(x)
$$

This emotional choice determines the emotions one receive, which in turn determines the magnitude of one's next reaction. I mentioned that the function that determines the magnitude of the reaction from the received emotion is determined by the following function in the case of an individual.

$$
F(x)=\frac{1}{\sqrt{2 \pi}} e^{-\frac{x^{2}}{2}}
$$

Here, equation ( 2) expresses the magnitude of the reaction. I have not modeled how one reacts.

I think that how an individual reacts is automatically determined by what happens and what emotions one receives. In other words, once the events and emotions are decided, the individual reaction is shown like a dictionary. Therefore, the individual reaction will be determined by how the events and emotions are determined.

Here, the event cannot be influenced by oneself. I don't think individuals can influence what has happened. I don't think an individual can influence an event, whether given by others, from nature, or as one's own thoughts. From my own experience, I thought this way.

I believe that an individual can influence whether one receives positive or negative emotions from an event. In other words, what determines one's reaction is whether one chooses a positive or negative sign of emotion. I thought that choosing the sign of emotion for event $X$ was the basis of an individual's character.

So how do individuals choose their emotions?

From my own experience, I thought that individuals would aim for $x=0$ for emotion $x$. $x=0$ is a convincing state for a series of events. In other words, a state of conviction is a state of stability, security, and emotionlessness. Toward that, the individual thought that the sum $X$ of the values of the events gradually approached $\frac{1}{\sqrt{2 \pi}}$ by the chain of 
the events or their reactions and the reception of emotions. I think that the sign of emotions at that time will be randomly determined so that you can be convinced.

In other words, one continuously thinks that "this event has such a positive side and such a negative side", and each time one receives emotions, a new reaction is generated from the reaction of the other party to one's reaction and one's own thought. That new reaction is added to the sum of the values of the events, and the next emotion is received. These flows continue until the one's emotion $x$ becomes $x=0$.

I consider this to be an individual's emotions choice and character. I think that the choice of emotional sign for the beginning event is the one that shows the individual tendency, but after that, I assumed that it was random.

\subsection{How to choose personal emotions}

Character was defined in Section 2.1. Think of this in the opposite direction. If you trace the result from $x=0$ to the beginning emotion in the opposite direction, you can see that it is a one-dimensional random walk as it is. In other words, the discussion of human emotion processing (Yabu, 2020) can be used as it is. The ratio of emotion selection by individual character, that is, the probability density function $s(x)$ for selecting the value of that emotion, is expressed by the following formula as in human emotion processing. I define this as a character function.

$$
s(x)=\frac{1}{\sqrt{2 \pi}} e^{-\frac{x^{2}}{2}}
$$

That is, the choice of emotions according to an individual's character is represented by a standard normal distribution.

It has been thought that the character of an individual is very different. It is also said that that is the individuality. However, it is only the difference in the reaction that is shown on the surface, and the character of the individual does not change from person to person. The character of the popular person and the character of the disliked person are basically the same, and the difference is in how they react from events and emotions.

No, maybe the events, emotions, and reactions don't match? I think there is a question of course. For example, when a boy's elementary school student is mean to a girl's elementary school student who he likes. Saying "I like her, but I'm mean to her" doesn't seem to match events, emotions, and reactions.

I think this is because he is not ready to receive the right feelings for the event, that is, his character isn't ready for the event. He doesn't know what kind of emotions he should choose for an event. He doesn't understand, so he thinks it's a negative emotion because 
it's an incomprehensible emotion. Therefore, he is mean to her as a reaction because he received a negative emotion.

Similar things can happen in adults. For example, your reaction to an event may be confusing and you may have frequent reactions that you do not understand. I've done something very rude, I have to do something to get this place, but my emotion processing can't keep up and I can't understand. This is an incomprehensible emotion, so it must be a negative emotion, so I have to apologize. So, I apologize. However, the other party is reacting with a confused expression. Why? It is an event that cannot be understood. Therefore, if an emotion that cannot be understood occurs again and a negative loop occurs, the total $X$ of the events will exceed $\frac{1}{\sqrt{2 \pi}}$ by repeatedly apologizing in a loud voice, "I'm sorry. I beg your pardon." This is a common situation for me. When this happens, my emotion $x$ never becomes $x=0$, so the other party continues to be confused and, in the end, "What does this person care about? I was apologized for the time, and I was given a very big reaction $X>\frac{1}{\sqrt{2 \pi}}$, and it was troublesome to worry about each one-dimensional random walk. This person can be seen below, " the other party thinks. I wrote this situation in the previous paper (Yabu, 2020). It can be said that this is also a situation in which the character of the individual does not work well and the character of the individual is immature.

3. About the character of the group

Now, regarding the character of an individual, it was found that the background of emotional selection is the equation ( 3 ), which is the same probability density function regardless of the individual. Individuals respond differently, but the basis is the same.

Therefore, regarding the character of a group of individuals, that is, what kind of emotions are selected as a group, the same probability density function as that of an individual is used, as in the discussion in the previous paper (Yabu, 2020). According to the following formula. This is the character function of the group.

$$
\operatorname{s}_{\text {group }}(x)=\frac{1}{\sqrt{2 \pi}} e^{-\frac{x^{2}}{2}}
$$

In other words, individuals and groups have the same character. In this sense as well, one doesn't have to think about one's own boundaries. This is because individuals and groups determine the sign of emotions according to the same probability density function. The graph of equation (4) is as follows. 


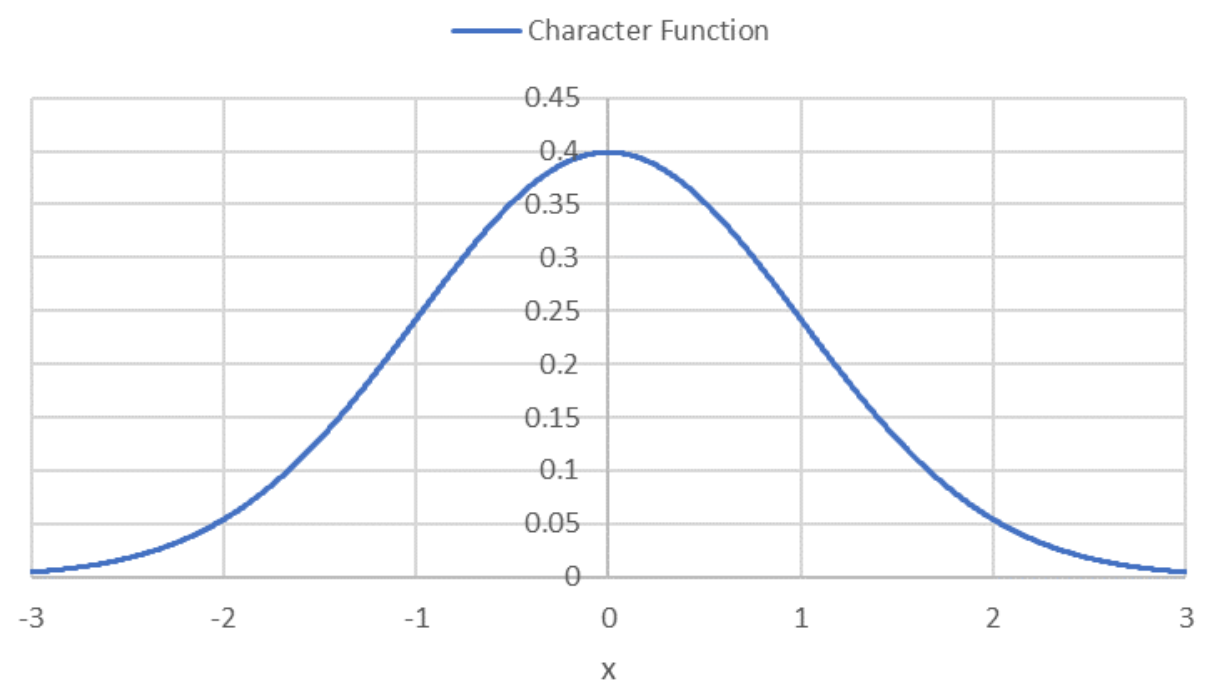

Figure 2 Individual and group character function

Also, it can be seen that the reaction shown as a group, not the magnitude of the reaction, is the average value of the reactions shown by individuals. Since the character of an individual and a group is expressed by the same probability density function. And the reaction is determined from events and emotions in an individual, like a dictionary. So, the same thing can be said for a group, and as a result, the group's control can be taken. I think that the mathematical model of the character presented can explain the reality. It is thought that the reaction shown as a group is likely to reveal the cultural aspect of the group.

4. About modeling with a one-dimensional random walk

So far, I have created and considered mathematical models for the character of individuals and groups. For a certain event $X$, select one's emotion $x$, and receive a new one's emotion $x$ from the reaction of one's own thought as a reaction accompanying it or the reaction of the other party when one's reaction occurs. I defined the character of the individual as the process of selecting the emotion $x$ that receives the series of these flows so that $x=0$. As for the result obtained from it, as already mentioned, the character function of an individual has a standard normal distribution, and the character function of a group of individuals also has a standard normal distribution.

In other words, if we remove the superficial part of the reaction, every human being has the same character function. The only difference is how one reacts.

I think some people say that my graph should not be as shown in Figure 2 because it is easy to think negatively. In the case of a one-dimensional random walk, it has the 
property that the probability of being in one of the positive and negative regions is greater than the probability of being in each of them. Therefore, there are people who are likely to receive negative emotions and people who are likely to receive positive emotions. Therefore, it is easy to think negatively, so it is not impossible to model with a one-dimensional random walk. Also, even people who are prone to receive negative emotions get positive emotions when they react according to the negative emotions and receive the expected reaction from the other person or themselves. In other words, even people who are prone to receive negative emotions may receive positive emotions momentarily.

From these points of view, it is appropriate to model an individual's character with a one-dimensional random walk.

5. How to deal with your character

Again, in my mathematical model, if we remove the superficial part of the reaction, then every human being has the same character function. The only difference is how one reacts. Both popular and disliked people have the same character function.

Therefore, when you face your character and want to correct your character, you use the fact that your character appears in the reaction, not your character. You will think about changing the reaction, that is shown when the event $X$ and emotion $x$ are determined like a dictionary, to the reaction you like. Individuals cannot influence the reactions that have occurred, but I think that individuals can change how they react. The character function of an individual is statistically determined and is the same for everyone. You can't fix it. Rather, you don't have to fix it. Is it easy to think negatively? Do I have to think positively? People with negative thoughts and people with positive thoughts have the same character function after all. It's statistically determined, so you can't fix it yourself.

Therefore, you will think about changing the reaction, that is shown when the event $\mathrm{X}$ and emotion $\mathrm{x}$ are determined like a dictionary, to the reaction you like. You dare to avoid the reaction that was supposed to determine event $X$ and emotion $x$ as before. When that happens, you will accumulate the experience that problems do not actually occur. By doing so, you will gradually change the reaction that was supposed to determine event $X$ and emotion $x$ to a reaction that you think is preferable to me. Doing so will fix the reaction.

Repeat this for various combinations of events $X$, emotions $x$, and reactions that you do not like. By doing so, I think that the superficial reaction will change to something that is favorable to you. 
That's how I think to deal with my character. I also practice. I will report any changes.

\section{Conclusion}

I considered the human character. I defined the character of the individual as the process of selecting the emotion $x$ that receives the series of flows so that $x=0$. The flows mean that for a certain event $\mathrm{X}$, select one's emotion $\mathrm{x}$, and receive a new one's emotion $\mathrm{x}$ from the reaction of one's own thought as a reaction accompanying it or the reaction of the other party when one's reaction occurs. $x=0$ means a situation that is convincing, reassuring, stable, and emotionless. I also defined that what kind of reaction an individual reacts to, not the magnitude of an individual's reaction, is shown like a dictionary when event $X$ and emotion $x$ are determined. Based on this, I have shown that when modeled using a one-dimensional random walk, the character function, which is a probability density function of how much an individual selects an emotion, can be modeled with a standard normal distribution. It also showed that the character function of a group of individuals can also be represented by a standard normal distribution. This shows that the group can take control because the character functions of the individual and the group are the same. Finally, even people who are easy to think negatively can model with a one-dimensional random walk, and since their character function is statistically determined and cannot be changed, I proposed a way that change their character by changing the reaction they show. I will try it myself. I will report any progress.

It would be an unexpected joy if it helps.

\section{References}

Yabu, T. (2020). Mathematical approach to human emotion processing. Retrieved from psyarxiv.com $/ \mathrm{k} 3 \mathrm{j} 4 \mathrm{z}$ 